NASA/CR-2002-211923

ICASE Report No. 2002-33

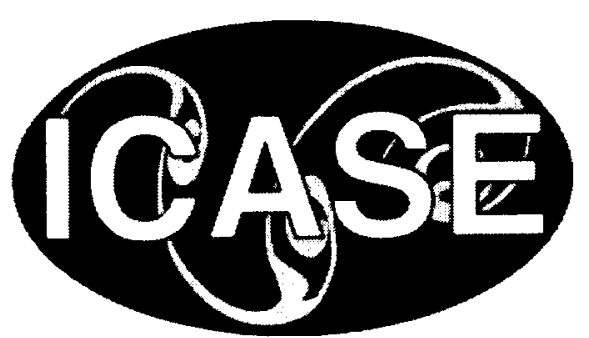

\title{
Termination of String Rewriting Rules that have One Pair of Overlaps
}

Alfons Geser

ICASE, Hampton, Virginia 


\section{The NASA STI Program Office ... in Profile}

Since its founding, NASA has been dedicated to the advancement of aeronautics and space science. The NASA Scientific and Technical Information (STI) Program Office plays a key part in helping NASA maintain this important role.

The NASA STI Program Office is operated by Langley Research Center, the lead center for NASA's scientific and technical information. The NASA STI Program Office provides access to the NASA STI Database, the largest collection of aeronautical and space science STI in the world. The Program Office is also NASA's institutional mechanism for disseminating the results of its research and development activities. These results are published by NASA in the NASA STI Report Series, which includes the following report types:

- TECHNICAL PUBLICATION. Reports of completed research or a major significant phase of research that present the results of NASA programs and include extensive data or theoretical analysis. Includes compilations of significant scientific and technical data and information deemed to be of continuing reference value. NASA's counterpart of peer-reviewed formal professional papers, but having less stringent limitations on manuscript length and extent of graphic presentations.

- TECHNICAL MEMORANDUM. Scientific and technical findings that are preliminary or of specialized interest, e.g., quick release reports, working papers, and bibliographies that contain minimal annotation. Does not contain extensive analysis.

- CONTRACTOR REPORT. Scientific and technical findings by NASA-sponsored contractors and grantees.
- CONFERENCE PUBLICATIONS. Collected papers from scientific and technical conferences, symposia, seminars, or other meetings sponsored or cosponsored by NASA.

- SPECIAL PUBLICATION. Scientific, technical, or historical information from NASA programs, projects, and missions, often concerned with subjects having substantial public interest.

- TECHNICAL TRANSLATION. Englishlanguage translations of foreign scientific and technical material pertinent to NASA`s mission.

Specialized services that complement the STI Program Office's diverse offerings include creating custom thesauri, building customized data bases, organizing and publishing research results ... even providing videos.

For more information about the NASA STI Program Office, see the following:

- Access the NASA STI Program Home Page at http://www.sti.nasa.gov

- Email your question via the Internet to help@sti.nasa.gov

- Fax your question to the NASA STI Help Desk at (301) 621-0134

- Telephone the NASA STI Help Desk at (301) 621-0390

- Write to:

NASA STI Help Desk

NASA Center for AeroSpace Information 7121 Standard Drive Hanover, MD 21076-1320 
NASA/CR-2002-211923

ICASE Report No. 2002-33

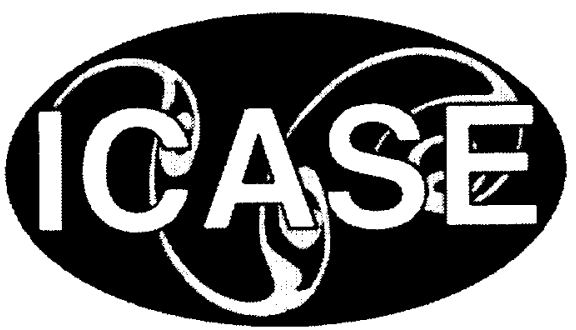

\section{Termination of String Rewriting Rules that have One Pair of Overlaps}

Alfons Geser

ICASE, Hampton, Virginia

ICASE

NASA Langley Research Center

Hampton, Virginia

Operated by Universities Space Research Association 
Available from the following:

NASA Center for AeroSpace Information (CASI)

7121 Standard Drive

Hanover, MD 21076-1320

(301) 621.0390
National Technical Information Service (NTIS) 5285 Port Royal Road

Springfield, VA 22161-2171

(703) 487-4650 


\title{
TERMINATION OF STRING REWRITING RULES THAT HAVE ONE PAIR OF OVERL APS*
}

\author{
MHONS GLSLR'
}

\begin{abstract}
This paper presents a partial solution to the long standing open problem of termination of one-rule string rewriting. Overlaps between the two sides of the rule play a central role in exist ing termination criteria. We characterize termination of all one-rule string rewriting systems that have one such orerlap at cither etde. This both completes a result of Kurth and generalizes a result of Shikishima-Tsuji et al.
\end{abstract}

Key words. scmi-Thue system, string lewriting, one-rule, single-mule, temination, miform termination, overlap

Subject classification. (omputer Scince

1. Introduction and Related Work. Termination of one-rule string rewriting systems (SRSs) is a long standing open problem $[12,13.11,15,14.7,16,18,2,3,4]$. The fir st systematic approach wals started by kurth [8]. He introduced a number of termination criteria to solve termination for all $t \rightarrow r$ where $|r| \leq 6{ }^{1}$

Most of hurth's criteria ( 5 out of 8 ), and indeed most of the criteria introduced since. are based on two sets: the set of overlaps of the left hand side (from the left end) with the right hand side (from the right end); and the set of overlaps of the right hand side (from the left end) with the left hand side (from the right end). Kurth's Criterion D states that we have termination if one or both of the two sets are cmpty.

In the case where both sets are singletons, we say that the one-rule SRS has one pair of onerlaps. Kurth [8] provides Criterion $F$ specifically for this case. As Criterion $F$ ran only prove termination of rules that are left barren or right barren, it is incomplete as we will show (Exauple 2). Shikishima-Tsuji ot al. [16. Theorem 2] show that a confluent one-rule SRS with one pair of overlap:s terminates if and only if there are no loops of lengthe 1 or 2 . As a consecuence termination of such SRSs is decidable.

This paper completely solves the termination problem for one-rule SliSs with one overlap pair. We prove that such an SRS terminates if and only if it has no loop of lengths 1,2 or 3 (Theorem 7.1). This implies decidability of the termination problem.

It turns out that the extension is non-trivial. There are two behaviours that were observed neither by Kurth nor by Shikishima-Tsuji et al. Loops of length 3 is one of them; the other is terminating non-tame rules.

This paper makes the following original contributions:

1. Termination of one-rule SRSs with one overlap pair is shown de idable.

2. Termination of one-rule SRSs with one overlap pair is shown equ valent to the non-existence of loops of length 3 or lesss.

3. Terminating one-rule SRSs with one overlap pair are shown to have lincar derivation lengths.

4. The first termination criterion for a class of non-tame one-rule siRSs.

*This work was supported be the National Aeronalutes and Space Administration under NASA Contract No. NAS1-970l6

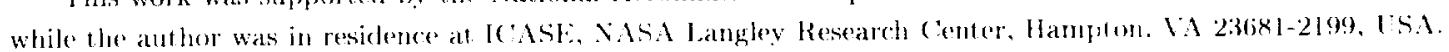

†Address: ICASt, Mail Stop 1326 NASA Langley Reserch center, Hampton, V. 23681. E-mail: geser@icase.edu

'An English presentation of Kurth's chapter on termination can be found in the auther's habilitation thesis [3]. 
The paper is organized as follows. After the preliminaries (Section 2) and an introduction to left barren and tame rules (Section 3). we focus on the interesting non-tame case. In Section 4, we derive a pattern that describes the non-tane' rules. In Sections 5 and 6 , we solve the non-terminating and terminating non-tame rules, resperetively: Section 7 finally shows the main theorem of the palper and its ramifications.

2. Preliminaries. A string reuriting rule is a pair $l \rightarrow r$ of strings, $l, r \in \Sigma^{*}$ where $\Sigma$ is a given alphabet. A set of string rewriting rules is called a string rewriting system (SRS). An SRS $R$ induces a reurite step relation $\rightarrow$ defined by $s \rightarrow t$ if there are $u, v \in \Sigma^{*}$ and a rule $\ell \rightarrow r$ in $R$ such that $s=u(v$ and $t=u r u$. The SRS $R$ is said to terminate if there is no infinite scequenee of rewrite steps s, $s_{1} \rightarrow s_{2} \rightarrow \ldots$

A string $u$ is called a factor of $v$ if $v=$ sut for some $s, t \in \Sigma^{*}$; a prefix if $v=u t$ for some $t \in \Sigma^{*}$; a suffix if $v^{\prime}=$ su for some $s \in \mathbf{L}^{*}$. The prefix or suffix $u$ of $v$ is called proper if $u \neq v$. The set of overlaps of a string $"$ with a string $v$ is defined by

$$
\operatorname{OVL}(u, v)=\left\{u \in \Sigma^{+} \mid u=u^{\prime} w, v=u^{\prime}, u^{\prime} v^{\prime} \neq \varepsilon, u^{\prime} \cdot v^{\prime} \in \Sigma^{*}\right\} .
$$

The length of a string $u$ is denoted by $|u|$.

3. Left Barren Rules. For a fixed one-rule SRS $\{\ell \rightarrow r\}$ let $A=O$ OVL $(r, \ell)$ and $B=O$ OV $(\ell, r)$. In what follows we consider $A$ and $B$ as disjoint. For all $\alpha \in A$, the strings $\ell_{a}$ and $r_{a}$ are defined by $\ell=\alpha \ell_{n}$ and $r=r_{a} \alpha$, respectively. Likewise, for all $\beta \in B$, the strings $\ell_{;}$and $r_{i}$ are defined by $f=\ell_{3} \beta$ and $r=3 r_{3}$, respertively.

The following definition of "left barren" is after MeNaughton's corrected version. The original definition is renamed to "left s-barren" (see Definition 3.4), following a suggestion of Kobayashi ot al. [7].

DLFInITION 3.1 (Left harren, right barren [12]). A one-rule SRS $\{\ell \rightarrow r\}$ is called left barren if $\ell$ is not a factor of $r$ and no $\ell_{o}, \alpha \in A$ is a prefix of any concatenation $r_{i_{1}} \ldots r_{\beta_{k}}$ where $\beta_{1} \ldots \ldots \beta_{k} \in B . k \geq 1$. Dually, $\{\ell \rightarrow r\}$ is called right barren if $\ell$ is not a factor of $r$ and no $\ell_{\beta}, \beta \in B$ is a suffix of any concatenation $r_{r_{1}} \ldots r_{\alpha_{k}}$ where $\alpha_{1}, \ldots, \alpha_{k} \in A, k \geq 1$.

A one-rule SRS $\{\ell \rightarrow r\}$ is called non-overlapping if $O V \mathrm{~L}(\ell, \ell)=\emptyset$.

Tнговем 3.2 ([12]). Every non-overlapping, left barren, one-rule SRS terminates.

THEOREM $3.3([3])$. Every left barren one-rule SRS terminates.

By symmetry w.r.t. reversal of strings also every right barren one-rule SRS terminates.

Definition 3.4 (Left s-barren, right s-barren $[12,7]$ ). A rule $\ell \rightarrow r$ is called left s-barren if no $\ell_{\alpha}, \alpha \in A$ is a prefix of any $r_{\beta}, \beta \in B$. Dually $\ell \rightarrow r$ is called right s-barren if no $\ell_{\beta}, \beta \in B$ is a suffix of any $r_{\alpha}, \alpha \in A$.

A left barren rule is left s-barren, but the converse usually does not hold. Indeed we will encounter left s-barren, not left barren rules later in this paper. They belong to a class of rules whose termination is particularly difficult to show. Next we will define this class.

In the following definition we consider $A, B$ as (disjoint) alphabets. For $\bar{\alpha}=\alpha_{1} \alpha_{2} \ldots \alpha_{k} \in A^{*}$ we define $\ell_{\bar{i}}$ by $\ell_{\bar{a}}=\ell_{\alpha_{1}} \ell_{\alpha_{2}} \ldots \ell_{\alpha_{k}}$. And dually, for $\bar{\beta}=\beta_{1} \beta_{2} \ldots \beta_{k} \in B^{*}$ we define $\ell_{3}$ by $\ell_{\bar{\beta}}=\ell_{\beta_{1}} \ell_{\beta_{2}} \ldots \ell_{\beta_{k}}$.

Kobayashi et al. [7] introduced the notion of tame, non-overlapping one-rule SRSs.

Drfinition 3.5 (Tamo [3]). Let $\{\ell \rightarrow r\}$ be a one-rule SRS. The sets $C$ and $D$ are defined by

$$
\begin{aligned}
& C=\left\{r^{\prime} \in \Sigma^{*} \mid r=\beta \ell_{\bar{\alpha}} r^{\prime}, \beta \in B, \bar{\alpha} \in A^{*}\right\}, \\
& D=\left\{r^{\prime} \in \Sigma^{*} \mid r=r^{\prime} \ell_{\bar{a}} \alpha, \alpha \in A, \bar{\beta} \in B^{*}\right\} .
\end{aligned}
$$

Then $t \rightarrow r$ is called tame if $\ell$ is neither of the form

$$
\alpha r_{1} r_{2} \ldots r_{k} w \text {, }
$$


for any a $\in A, k \geq 1, r_{1}, \ldots, r_{k} \in C$, and non-empty prefix w of an rlement of $C:$ nor of the form

$$
u r_{1} r_{2} \ldots r_{j} \beta
$$

for any $\beta \in B, j \geq 1, r_{1}, \ldots, r_{j} \in D$. and non-empty suffix w of an clement of $D$.

The following result is implicit in Kobayashi ot al. [7. Cor. 5.9].

THEOREM 3.6. Every non-overlapping. tame, left s-barren one-rule SRS is left barren.

TH:oreal 3.7 ([3]). Every tame, left s-burven one-rule SRS is left barren.

By symmetry erery tame, right s-barren one-ruke SRS is right harre.t.

Proof. For a proof by contradiction, assume that $f \rightarrow r$ is not left barren, i.e., some to is a prefix of some concatenation $r_{i i_{1}} r_{i j_{2}} \cdots r_{i_{n}}$. Let $n$ be minimal. If $n=1$ then $r \rightarrow r$ is not left s-barren. So $n \geq 2$

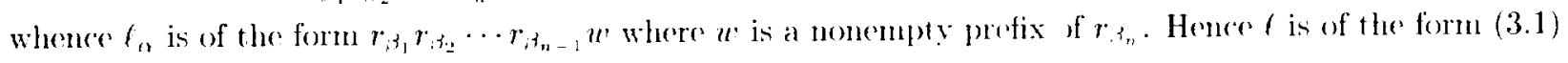
and so $f \rightarrow r$ is not tame.

4. A Reduction of the Problem. Throughout the remainder of this paper we assume a one-rule SRS $\{f \rightarrow r\}$ that has one pair of overlaps, i.e.. $\mid$ OVL $(r, f)|=| O V L(f, r) \mid=1$. Let then $a . \beta \in \Sigma^{+}$bo detined by OVL $(r, \ell)=\{\alpha\}$ and $O \mathrm{VL}(\ell, r)=\{\Leftrightarrow\}$.

We will devote the greater part of the paper to solving the interesting case: rules that are left s-barren but neither left barren nor right s-barren. According to Theorem 3.T. th ese are non-tame. specifically they are of the form (3.1). In this section we will derive the general patter' of such rules. Let us henceferth assume that $\ell$ is not a factor of $r$ and that $|\ell|<|r|$.

The first pattern is derived without the right-s-barren hypothesis.

Leman 4.1. Let $\ell \rightarrow r$ be left s-barren but not left barren. Then $|\beta|>|a|$ and $\ell \rightarrow r$ is of the form

$$
\alpha\left(u w^{\prime}\right)^{n-1} w \rightarrow B w w^{\prime}
$$

for some $n \geq 2, u^{\prime} \in \Sigma^{*}$, and $w \in \Sigma^{+}$.

Proof. Let $\ell \rightarrow r$ be left s-barren but not left barren. Then we get $1 y$ the respective definitions that $\ell_{a}$ is not a prefix of $r_{3}$ and that $\ell_{a}$ is a prefix of $r_{3}^{n}$ form some $n \geq 1$. Hence $r_{3}$ is a proper prefix of $\ell_{a}$. So let $\ell_{a}=r_{i,}^{\prime \prime-1} w$ where $n \geq 2$, and $w$ is a non-empty prefix of $r_{3}$. Let $w^{\prime} \in \Sigma^{*}$ be defined by $r_{s}=w^{\prime} w^{\prime}$. By back-substitution we get the form (4.1). From $\left|\beta r_{;}\right|=|r|>|f|=\left|\alpha r_{3}^{m-1} w\right|$ we conclude $|\beta|>|\alpha|$.

If we ald the right-s-barren hypothesis, then we can rule out the ca we where $\alpha$ and $\beta$ overlap in $t$.

LemMA 4.2. If $f \rightarrow r$ is left s-barren but neither left barren nor right s-barren, then $|a|+|3| \leq|\ell|$.

Proof. For a proof by contradiction assume $|\alpha|+|\beta|>|\ell|$. Let $\ell \rightarrow r$ be left s-barren but not left barren. By Lemma 4.1 we get that $\ell \rightarrow r$ has the form (4.1). Then $b y|\alpha|+|\beta|>|\ell|$ there is a non-empty suffix $u$ of $\alpha$ such that $\beta=u\left(w w^{\prime}\right)^{n-1} w$. Define $\alpha^{\prime} \in \Sigma^{*}$ by $a=\alpha^{\prime} u$. The string $\alpha^{\prime}$ is non-empty by $\beta \neq \ell$. Thus $\ell$ and $r$ are of the form

$$
\begin{aligned}
& \ell=\alpha^{\prime} u\left(w w^{\prime}\right)^{n-1} w \\
& r=u\left(w w^{\prime}\right)^{n-1} w w w^{\prime}
\end{aligned}
$$

for some $n \geq 2, w^{\prime} \in \Sigma^{*}$, and $\alpha^{\prime}, u, w \in \Sigma^{+}$.

Now let morever $f \rightarrow r$ not be right s-barren, i.e.. let $f_{3,3}$ be a suffix of $r_{0}$. This is expressed equivalently by the string equation $z f_{3} \alpha=r$ for some $z \in \Sigma^{*}$. Using $f_{3}=\alpha^{\prime}$ this inslantiates to

$$
z \alpha^{\prime} \alpha^{\prime} u=u\left(w w^{\prime}\right)^{n-1} w w w^{\prime}
$$


Let $m \geq 0$ be maximal such that $\left(\left(w w^{\prime}\right)^{n-1} w^{\prime} w^{\prime} w^{\prime}\right)^{m}$ is a suftix of $u$. Define $u_{1} \in \Sigma^{*}$ by $u=u_{1}\left(\left(w w^{\prime}\right)^{n-1} w w w^{\prime}\right)^{m}$. Then $u_{1}$ is a proper suffix of $\left(w w^{\prime}\right)^{n-1} w w w^{\prime}$, and the equation reduces to $z \alpha^{\prime} \alpha^{\prime} u_{1}=u_{1}\left(w w^{\prime}\right)^{n-1} w w^{\prime} w^{\prime}$. If $m>0$ then $a^{\prime} u_{1} \in O \mathrm{OL}(r, 0)$, a contradiction. So $m=0$ and $u=u_{1}$.

If $u_{1}$ is a suffix of $w w^{\prime}$ then $u_{1} w \in O \mathrm{OVL}(f, r)$, a contradiction. So $w u^{\prime}$ is a proper sutfix of $u_{1}$. Let $u_{2} \in \Sigma^{+} b_{0}$ defined by $u_{1}=u_{2} w w^{\prime}$. The equation reduces to $z \alpha^{\prime} \alpha^{\prime} u_{2}=u_{2}\left(u w^{\prime}\right)^{\prime \prime} w^{\prime}$.

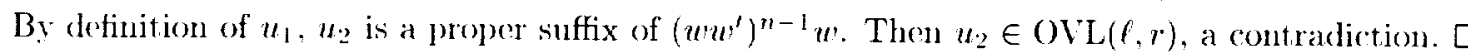

If $a$ and $\beta$ do not overlap in 6 , then wo can narrow the pattern for the rule:

LAMM 4.3. Let $\rightarrow r$ be left s-barren but not left barren. If $|\alpha|+|\beta| \leq|f|$ then $\ell \rightarrow r$ is of the form

$$
\text { кuryow } \rightarrow \text { yow'uxyo }
$$

for some $x \in \Sigma^{*}$ and $y, \alpha, w \in \Sigma^{+}$.

Proof. Let $f \rightarrow r$ be left s-barren but not left barren. By Lemma 4.1 we get that $t \rightarrow r$ has the form (4.1).

Case 1: $\beta=w^{\prime \prime}\left(w^{\prime} w^{\prime}\right)^{i}$ for some $0 \leq i \leq n-1$, and some non-empty suffix $u^{\prime \prime}$ of $a$. If $i \geq 1$ then $w^{\prime \prime} \in O \mathrm{VL}(t, r)$, a contradiction. So $i=0$ and $\beta=w^{\prime \prime}$. Then

$$
|r|-|\ell|=\left|u^{\prime \prime}\right|+|w|+\left|w^{\prime}\right|-\left(|\alpha|+n|w|+(n-1)\left|w^{\prime}\right|\right)<0,
$$

again a contradiction.

Case 2: $\beta=w^{\prime \prime} w\left(w^{\prime} w\right)^{\prime}$ for some $0 \leq i \leq n-2$, and some nonempty suffix $w^{\prime \prime}$ of $w^{\prime}$. If $i \geq 1$ then $w^{\prime \prime} w \in \mathrm{OVL}(\ell, r)$, a contradiction. So $i=0$ and $\beta=w^{\prime \prime} w$. Let $w^{\prime}=x u^{\prime \prime}$ for some string $x$. Then we have

$$
\begin{aligned}
& \ell=\alpha\left(w r w^{\prime \prime}\right)^{n-1} w, \\
& r=w^{\prime \prime} w w x u^{\prime \prime}
\end{aligned}
$$

and so

$$
\begin{aligned}
|r|-|f| & =2\left|w^{\prime \prime}\right|+2|w|+|x|-\left(|\alpha|+(n-1)\left|w^{\prime \prime}\right|+(n-1)|x|+n|w|\right) \\
& =(3-n)\left|w^{\prime \prime}\right|+(2-n)|w|+(2-n)|x|-|\alpha| .
\end{aligned}
$$

If $n \geq 3$ then $|r|-|\ell|<0$. So $n=2$ and $|r|-|\ell|=\left|w^{\prime \prime}\right|-|\alpha|>0$ whence $\left|w^{\prime \prime}\right|>|\alpha|$. By definition of $\alpha$ now $\alpha$ is a proper suffix of $w^{\prime \prime}$. Let $w^{\prime \prime}=y \alpha$ for some $y \in \Sigma^{+}$. We conclude that $\ell \rightarrow r$ is of the form (4.2).

Putting Lemma 4.2 and 4.3 together allows us to narrow the rule pattern further:

LEMMA 4.4. If $\ell \rightarrow r$ is left s-barren but neither left barren nor right s-barren then $\ell \rightarrow r$ is of the form.

$$
\alpha w x(y \alpha w x)^{m+1} \alpha w \rightarrow y \alpha w r x w x(y \alpha w x)^{m+1} \alpha .
$$

for some $m \geq 0, x \in \Sigma^{*}$, and $\alpha, w, y \in \Sigma^{+}$.

Proof. Let $\ell \rightarrow r$ be left s-barren but neither left barren nor right s-barren. By Lemma 4.2 we get $|\alpha|+|\beta| \leq|\ell|$. By Lemma 4.3 we get that $\ell \rightarrow r$ has the form (4.2).

The property that $\ell \rightarrow r$ is not right s-barren means that $\ell_{3}=\alpha w x$ is a suffix of $r_{a}=y a w w x y$. Then we have to solve the string equation

$$
z \alpha u x=y \alpha u w x y
$$

for $z, x \in \Sigma^{*}, \alpha, w, y \in \Sigma^{+}$.

Let $m \geq 0$ be maximal such that $y^{\prime \prime \prime}$ is a suffix of $x$. Define $x_{1} \in \Sigma^{*}$ by $x=x_{1} y^{\prime \prime \prime}$. Then $z$ ore $x_{1}=$ you'w $x_{1} y$ and $x_{1}$ is a proper suffix of $y$. Define $y_{1} \in \Sigma^{+}$by $y=y_{1} x_{1}$. Then $z \alpha w=y_{1} x_{1}$ owew $x_{1} y_{1}$. 
If $y_{1}$ is a suffix of $u^{\text {then }} y_{1} \in$ OVL( $(.$,$) , a contradictions. So w$ in a proper suftix of $y_{1}$. Define $y_{2} \in \Sigma^{+}$ by $y_{1}=y_{2} w$. Then the equation reduces to $z a=y_{2} w_{1} x_{1} a w^{2} x_{1} y_{2}$.

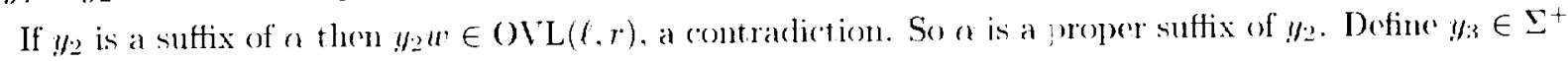

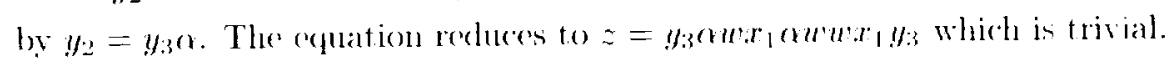

By bark-sulstitution we get

$$
\begin{aligned}
& y=y_{1} \cdot x_{1}=y_{2} u_{2} x_{1}=y_{3}\left(t+w_{1} x_{1} .\right. \\
& r=x_{1} y^{\prime \prime \prime}=x_{1}\left(y_{3} \cap a x_{1}\right)^{m} \text {. }
\end{aligned}
$$

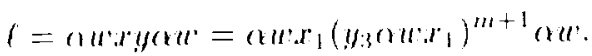

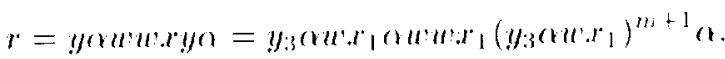

and thus the form (4.3) by the renaming $x_{1} \mapsto r, y: 3 \mapsto y$.

The following is interesting to note. It explains why rules of the form (4.3) were not observed by Shikishima-Tsuji ot al.

THiorien 4.5. All rules of the form (4.3) ate non-ronfluent.

Proof. A onc-rule SRS $\{\ell \rightarrow r\}$ where $|\ell|<|r|$ is confluent if and only if $O \mathcal{})(\ell, f) \subseteq O \mathcal{O L}(r, r)$ by a result

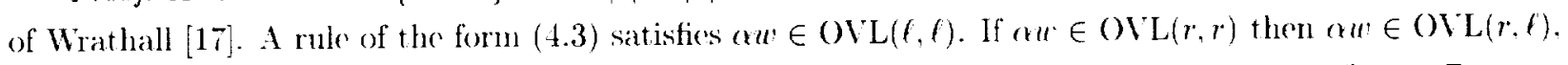
a contradiction to $O \mathrm{OL}(r, \ell)=\{\alpha\}$. So $\alpha u \in O \mathrm{OV} \mathrm{L}(\ell, \ell) \backslash O \vee \mathrm{L}(r, r)$ whence $\ell \rightarrow r$ is not confluent.

In the next two sections we are going to identify the non-terminating and the terminating instances of the form (4.3).

5. The Non-terminating Case. A rule of the form (4.3) loops in the following case:

LemMa 5.1. Let $\ell \rightarrow r$ be left s-barren but neither left barren nor ight s-barren. If $\ell_{3} f_{3}$ is a suffir of $r_{a}$. then the one-rule SRS $\{f \rightarrow r\}$ has a loop of length 3 .

Proof. Like in the proof of Lemma 4.1. we get $\ell_{\alpha}=r_{j}^{n-1} w$ and $r_{i 3}=w^{\prime} w^{\prime}$ for some $u \in \Sigma^{+}, w^{\prime} \in \Sigma^{*}, n \geq 2$. In the proof of Lemma 4.3 we showed $n=2$. With $r_{\alpha}=v \ell_{i 3} \ell_{;}$for some $v \in \Sigma^{*}$, we then get a loop:

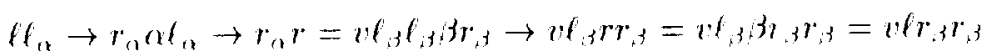

$$
\begin{aligned}
& =v \ell_{a} w^{\prime} \text {. }
\end{aligned}
$$

These loops are also instances of Kurth's criterion for loops of length 3 [9, Theorem 2. Case A]. The following little result provides an altornative criterion to Lemma 5.1 .

LemMA 5.2. If $t \rightarrow r$ has the form (4.3) then the following are equinalent:

1. $\ell_{i 3} f_{3}$ is a suffixix of $r_{\alpha}$,

2. $m=0$ and $y=y^{\prime}$ cuer for some $y^{\prime} \in \Sigma^{+}$.

Proof. Obviously (2) implies (1). Next we show the converse by contradiction. Let $\ell \rightarrow r$ have the form (4.3) and let $\ell_{3} \ell_{3}$ be a sulfix of $r_{0}$. Define $v \in \Sigma^{*}$ by $r_{\alpha}=v \ell_{3} \ell_{3}$. If $m>0$ then $y$ is a suffix of $y / \alpha e$ and then $y(x) \in$ OVL $(\ell, r)$, a contradiction. With $m>0$, the string cuer is a suffix of cruxuy. If $y$ is a suffix of $\alpha w x$ then $y \times w \in O \operatorname{OVL}(f, r)$, a contradiction. So $r m x$ is a prop cr suftix of $y$. i.e., there is $y^{\prime} \in \Sigma^{+}$ such that $y=y^{\prime}(a w x$.

EXAMl'L: 1. The one-rule SRS

$a b d a b a b a b \rightarrow d a b a b a b b d a b a b x$ 
has. a loop of length s:

$$
\begin{aligned}
& \text { abdebababbdababab } \rightarrow \\
& \text { dabababbdebababdababab } \rightarrow \\
& \text { dabababbdababrlabababdrababa } \rightarrow \\
& \text { dabababbdabdabababbdababab dababa. }
\end{aligned}
$$

Redexes are underlined. The re-oceurrence of the start string is indicated by a box. This example provides the smallest non-terminating witness $(n \mid=14)$ of Lemma 4.4 .

6. The Terminating Case. For this section let us assume a rule of the form (4.3) where $l_{3} t_{1}$ is not a suffix of $r_{0}$. We are going to reduer termination of such a rule to termination of an SRS $R$ ower a different aljhabet. Termination of $R$ will be asy to prove.

Detine $r_{\delta}, r_{i, \beta, \alpha}$, and $r_{i, \beta}$ by

$$
r=r_{\delta} \ell_{i \gamma \alpha} \alpha, \quad r=\beta r_{\beta, \alpha} \alpha, \quad r=\beta r_{\beta, \delta} \ell_{;} \alpha \alpha .
$$

These definitions are somul as witnessed by

$$
\begin{aligned}
\beta & =y \alpha w r \alpha w, \\
\ell_{\beta} & =\alpha w x(y \alpha w x)^{\prime \prime \prime}, \\
r_{\delta} & =y \alpha w x \alpha w w x y, \\
r_{i, \alpha} & =w x(y \alpha w x)^{m+1}, \\
r_{i, \delta} & =w x y .
\end{aligned}
$$

LlimaA 6.1. Let $\ell \rightarrow r$ have the form (4.3). Then the following rewrite steps exist:

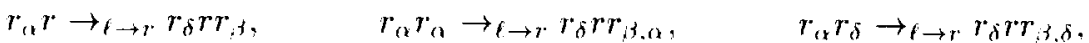

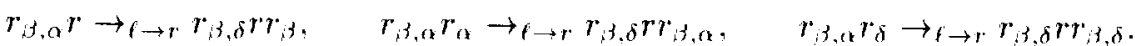

Proof. Routine.

LEMmA 6.2. Let $\ell \rightarrow r$ have the form (4.3) and let $f_{\beta} \ell_{, 3}$ not be a suffix of $r_{\alpha \gamma}$. Then $\ell$ is not a factor of any of the follouing: (1) $r_{\delta}^{i} r$, (2) $r r_{\beta}$, (3) $r r_{\beta, \delta} r_{\delta}^{i} r$ for any $i \geq 0$.

Proof. For Claim 1, lot $i \geq 1$ be least such that $\ell$ is a factor of $r_{\delta}^{i} r$. Then $\ell_{\beta}$ is a suffix of $r_{\delta}^{i}$ because $\beta$ is the only overlap of $\ell$ with $r$. Since $\ell_{B} \ell_{B}$ is not a suffix of $r_{\alpha}=r_{\delta} \ell_{3,}, \ell_{B 3}$ is not a suffix of $y$. Hence $y$ is a proper suffix of $\ell_{i}$ and so of $y \alpha w x$. So $y \alpha u, \in O V L(\ell, r)$, a contradiction.

For Claim 2, let $\ell$ be a factor of $r r_{\beta}$. Because $\alpha$ is the only overlap between $r$ and $\ell$, we have $\left|\ell_{\alpha}\right| \leq\left|r_{\beta}\right|$. a contradiction.

For Claim 3 assume that $\ell$ is a factor of $r r_{\beta, \delta} r_{\delta}^{i} r$ for some $i \geq 0$. By Claims 1 and $2, \ell$ is neither a factor of $r_{i, \delta} r_{\delta}^{i} r$ nor of $r r_{3, \delta}$; so $\ell$ is of the form $\ell^{\prime} r_{3, \delta} r_{\delta}^{j} \ell^{\prime \prime}$ for sone $0 \leq j \leq i$ and some non-empty suffix $\ell^{\prime}$ of $r$ and some non-empty prefix $\ell^{\prime \prime}$ of $r$. Thus $\ell$ is of the form $\alpha r_{i, \delta} r_{\delta}^{j} \beta$. If $j=0$ then $w x(y \alpha w x)^{m}=u x y$ which contradicts $y, \alpha \in \Sigma^{+}$. So $j>0$ and $y$ is a proper suffix of $\ell_{3}$. We get a contradiction by $y \alpha w \in O \operatorname{VL}(\ell, r)$.

The six-rule SRS $R$ over $\Omega=\{a, b, c, d, e, f\}$ is defined as follows:

$$
\begin{aligned}
R=\left\{g^{\prime} g^{\prime \prime} \rightarrow h^{\prime} f h^{\prime \prime} \mid\right. & \left(g^{\prime}, h^{\prime}\right) \in\{(a, d),(c, c)\}, \\
& \left.\left(g^{\prime \prime}, h^{\prime \prime}\right) \in\{(a, c),(d, c),(f, b)\}\right\}
\end{aligned}
$$


Define the weight $\omega^{*}(x)$ of a string $x$ by $w^{t}(a)=u t(c)=3, \operatorname{wt}(b)=u t(d)=u t(c)=u t(f)=1$, and $w t^{*}\left(x_{1} \ldots x_{k}\right)=\sum_{i=1}^{k} w t\left(x_{i}\right)$. Then $R$ terminates by

$$
w^{*}(u)-u^{\prime} f^{*}\left(u^{\prime}\right)=\left(w t\left(g^{\prime}\right)-w^{\prime}\left(h^{\prime}\right)\right)-w t(f)+\left(u t\left(g^{\prime \prime}\right)-w^{\prime}\left(h^{\prime \prime}\right)\right)=2-1+0>0
$$

for all rewrite steps $u \rightarrow_{R} u$.

Let the string homomorphism $\phi: \Omega^{*} \rightarrow \Sigma^{*}$ be defined $b y, \phi(a)=r_{0} . \phi(b)=r_{3 .} \phi(c)=r_{3, \alpha} . \phi(d)=$

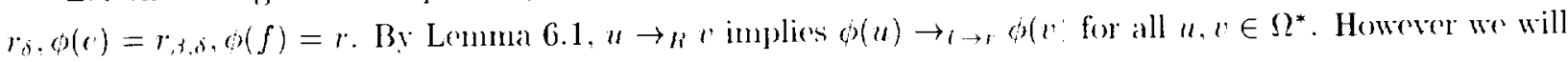
need the converse direstion. To this ond let us define the regular language. $M$ by

$$
\mathcal{H}=\left(a+d(f e)^{*}+d\left(f()^{*} f()^{*}\left(a f+d(f c)^{*} f(c f+b)\right)+f .\right.\right.
$$

Let $\phi[\mathcal{M}]$ denote the set $\{\phi(u) \mid u \in \mathcal{M}\}$. Wo are going to show that $\{l \rightarrow r\}$-reduction steps on $o[. \mathcal{M}]$ can be simulated by R-reduction steps. First we show that R-reduction preserves $\phi[. \mathcal{M}]$.

Lamind 6.3. If $u \in \mathcal{M}$ and $u \rightarrow n v$ then $v \in \mathcal{M}$.

Proof. Let $\left(g^{\prime}, h^{\prime}\right) \in\left\{(a, d),\left(c^{\prime}, c^{\prime}\right)\right\}$ and $\left(g^{\prime \prime}, h^{\prime \prime}\right) \in\{(a, c),(d, c) .(f . b)\}$. Let. $u=u^{\prime} g^{\prime} g^{\prime \prime} u^{\prime \prime} \in \mathcal{M}$ and $v=u^{\prime} h^{\prime} f h^{\prime \prime} u^{\prime \prime}$. Then we derive

$$
\begin{array}{ll}
u^{\prime} \in\left(a+d\left(f c^{\prime}+d\left(f c^{\prime}\right)^{*} f()^{*}\right.\right. & \text { if } ! \iota^{\prime}=a . \\
u^{\prime} \in\left(a+d(f)^{*}+d\left(f()^{*} f(c)^{*} d\left(f c^{\prime}\right)^{*} f\right.\right. & \text { if } ! \iota^{\prime}=c .
\end{array}
$$

Case 1: $g^{\prime \prime}=a$. If $g^{\prime}=a$ then $u^{\prime \prime} \in \mathcal{M}$ whence $v=u^{\prime} d f\left(u^{\prime \prime} \in \mathcal{A}\right.$. If $g^{\prime}=c$ then $u^{\prime \prime}=f$ whence $v=u^{\prime} \cdot f r u^{\prime \prime} \in \mathcal{M}$.

Casc 2: $g^{\prime \prime}=d$. Then

$$
\begin{aligned}
u^{\prime \prime} \in & \left((f c)^{*}+(f)^{*} f c\right)\left(a+d(f e)^{*}+d(f c)^{*} f c\right)^{*}\left(a f+d(f e)^{*} f(c f+b)\right) \\
& +(f c)^{*} f(c f+b) .
\end{aligned}
$$

If $g^{\prime}=a$ then $v=u^{\prime} d f \circ u^{\prime \prime} \in \mathcal{M}$. If $g^{\prime}=c$ then $v=u^{\prime} \cdot f e u^{\prime \prime} \in \mathcal{M}$.

Case 3: $g^{\prime \prime}=f$. If $g^{\prime}=a$ then $u^{\prime \prime}$ is the empty string and $v=u^{\prime} d f b u^{\prime \prime} \in \mathcal{M}$. If $g^{\prime}=c$ then $u^{\prime \prime}$ is again the empty string and $v=u^{\prime} e f b u^{\prime \prime} \in \mathcal{M}$.

Next we derive a few properties of $u \in \mathcal{M}$ if $\phi(u)$ contains a factor $t$.

LFMna 6.4. Let $u \in \mathcal{M}$ and $s^{\prime}, s^{\prime \prime} \in \Sigma^{*}$. If $\phi(u)=s^{\prime}\left(s^{\prime \prime}\right.$ then $u=u^{\prime} g^{\prime} g^{\prime \prime} u^{\prime \prime} .\left|\phi\left(u^{\prime}\right)\right| \leq\left|s^{\prime}\right|<\left|\phi\left(u^{\prime} g^{\prime}\right)\right|$. $\left|\phi\left(u^{\prime \prime}\right)\right| \leq\left|s^{\prime \prime}\right|<\left|\phi\left(g^{\prime \prime} u^{\prime \prime}\right)\right|$ for some $u^{\prime}, u^{\prime \prime} \in \Omega^{*}, g^{\prime} \in\{a, c\}, g^{\prime \prime} \in\{a, d . f\}$.

Proof. Suppose that $u \in \mathcal{M}, s^{\prime}, s^{\prime \prime} \in \mathrm{I}^{*}$, and $\phi(u)=s^{\prime} \ell s^{\prime \prime}$. Let $u^{\prime} \in \Omega^{*}$ be the longest prefix of $u$ such that $\left|\phi\left(u^{\prime}\right)\right| \leq\left|s^{\prime}\right|$. Let $u^{\prime \prime} \in \Omega^{*}$ be the longest suffix of $u$ such that $\left.\mid \phi \cdot u^{\prime \prime}\right)|\leq| s^{\prime \prime} \mid$. By $|\phi(u)|>\left|\phi\left(u^{\prime} u^{\prime \prime}\right)\right|$ there is $v \in \Sigma^{+}$such that $u=u^{\prime} v u^{\prime \prime}$. Define $t^{\prime}, t^{\prime \prime} \in \Sigma^{*}$ by $s^{\prime}=\phi\left(u^{\prime}\right) t^{\prime}$ and $s^{\prime \prime}=t^{\prime \prime} \phi\left(u^{\prime \prime}\right)$. Then

$$
\phi(u)=\phi\left(u^{\prime}\right) \phi(v) \phi\left(u^{\prime \prime}\right)=\phi\left(u^{\prime}\right) t^{\prime}\left(t^{\prime \prime} \phi\left(u^{\prime \prime}\right)\right.
$$

whence $\phi(v)=t^{\prime}\left(t^{\prime \prime}\right.$. The (ase $|v|=1$ implies that $\ell$ is a factor of $r$ so $|v| \geq 2$. We distinguish cases on the form of $v$.

Case 1: $v \in \Omega \Omega^{*}(a+c)(a+d+f) \Omega \Omega^{*}$. Let $g^{\prime} \in\{a, c\}, g^{\prime \prime} \in\{a, d, f\} . r^{\prime}, v^{\prime \prime} \in \Omega^{*}$, and let $v=v^{\prime} g^{\prime} g^{\prime \prime} r^{\prime \prime}$. Wo further distinguish cases whether $v^{\prime}, v^{\prime \prime}$ are empty strings or not.

Case 1.1: $\left|v^{\prime}\right|=\left|v^{\prime \prime}\right|=0$. Then $v=g^{\prime} g^{\prime \prime}$. By definition of $u^{\prime}$ we get $t^{\prime}|<| \phi\left(g^{\prime}\right) \mid$. By definition of $u^{\prime \prime}$ we get $\left|t^{\prime \prime}\right|<\left|\phi\left(g^{\prime \prime}\right)\right|$. The claim follows. 
Case 1.2: $\left|r^{\prime}\right|=0 .\left|r^{\prime \prime}\right|>0 . B y|r|>|r|$ and $\left|r_{n}\right|>|f|$ and $u \in \cdot \mathcal{M}$ we get $v \in\left(a+c^{\prime}\right) d^{+}(a+d+f)$. Let $v=v_{0} g_{0}$ for some $r_{0} \in(a+c) d^{+}$, and $g_{0} \in\{a, d, f\}$. Then there are $\ell^{\prime}, f^{\prime \prime} \in \Sigma^{+}$such that $\ell=\ell^{\prime} f^{\prime \prime}$, $\phi\left(r_{0}\right)=t^{\prime} f^{\prime}$, and $\phi\left(g_{0}\right)=f^{\prime \prime} t^{\prime \prime}$. Since $\phi\left(g_{0}\right)$ is a prefix of $r$, we obtain $\left.f^{\prime \prime} \in O\right) \mathcal{L}(f, r)$, so $f^{\prime \prime}=3$ and $\ell^{\prime}=\ell_{3}$.

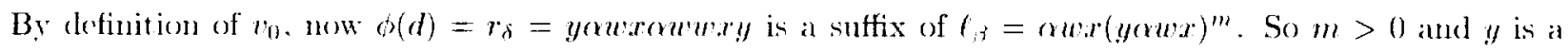

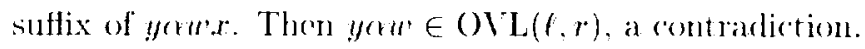

Case 1.3: $\left|e^{\prime}\right|>0 .\left|r^{\prime \prime}\right|=0$. Let $v=v_{0} g_{0}$ for some $r_{0} \in \Omega^{+}(a+r)$, and $g_{0} \in\{a, d, f\}$. Then there are $f^{\prime}, \ell^{\prime \prime} \in \Sigma^{+}$such that $\left.\ell^{\prime}=\ell^{\prime} f^{\prime \prime}, \phi\left(l^{\prime}\right)\right)=f^{\prime} f^{\prime}$, and $\phi^{\prime}\left(g_{0}\right)=f^{\prime \prime} t^{\prime \prime}$. Since $\phi\left(g_{0}\right)$ is a prefix of $r$. we (o)tain $f^{\prime \prime} \in O \mathrm{OVL}(f, r)$, so $f^{\prime \prime}=3$ and $f^{\prime \prime}=\ell$, . Then

$$
\left|\ell_{, 3}\right|=\left|\phi\left(x_{0}\right)\right|>|\phi(r)|=\left|r_{i, a 1}\right|>\left|\ell_{3}\right| \text {. }
$$

a contradiction.

Case 1.4: $\left|v^{\prime}\right|,\left|v^{\prime \prime}\right|>0$. By $|r|>|f|$ and $\left|r_{\alpha}\right|>|f|$ and $u \in \mathcal{M}$ we got $g^{\prime}=c$ and $g^{\prime \prime}=d$. So $\phi(c r d)=r_{i, a} r_{\delta}$ is a factor of $\ell$, whence $\left|r_{3, \alpha} r_{i}\right| \leq|\ell|$, a contradiction.

Case $2: v \in \Omega^{+} \backslash \Omega^{*}(a+c)(a+d+f) \Omega^{*}$. Define the set of fragments $\mathcal{F}(z)$ of a string $z \in \Omega^{*}$ as follows. If $z \in(\Omega \backslash\{f\})^{*}$ then $\mathcal{F}(z)=\{z\}$. Else $z=z_{10} f z_{1} \ldots f z_{n}$ for some $n \geq 1$ and unicue $z_{1}, \ldots, z_{n} \in(\Omega \backslash\{f\})^{*}$; then

$$
\mathcal{F}(z)=\left\{z_{1} f, f z_{2} f, \ldots, f z_{n-1} f, f z_{n}\right\}
$$

From $u \in \mathcal{M}$ then

$$
\mathcal{F}(u) \in(a+d)^{*} f+f(e+r)(a+d)^{*} f+f b .
$$

Because $|r|>|\ell|$, and $\ell$ is not a factor of $r$, we obtain $v \in \mathcal{F}(u)$. So

$$
v \in \mathcal{F}(u) \backslash \Omega^{*}(a+c)(a+d+f) \Omega^{*}=d^{*} f+f d^{*} f+f b .
$$

By Lemma $6.2, \phi(v)$ has no factor $\ell$, so this case is void.

Now we are ready to state the simulation lemma.

Lemma 6.5. Let $u \in \mathcal{M}$ and $t \in \Sigma^{*}$. If $\phi(u) \rightarrow_{\ell \rightarrow r} t$ then $\phi(v)=t$ and $u \rightarrow_{R}$ v for some $v \in \mathcal{M}$.

Proof. Let $u \in \mathcal{M}$ and $s^{\prime}, s^{\prime \prime}, t \in \Sigma^{*}$, and let $\phi(u)=s^{\prime} \ell s^{\prime \prime}$ and $t=s^{\prime} r s^{\prime \prime}$. By Lemma 6.4 there are $u^{\prime}, u^{\prime \prime} \in \Omega^{*}, g^{\prime} \in\{a, c\}, g^{\prime \prime} \in\{a, d, f\}$ such that $u=u^{\prime} g^{\prime} g^{\prime \prime} u^{\prime \prime}$ and $\left|\phi\left(u^{\prime}\right)\right| \leq\left|s^{\prime}\right|<\left|\phi\left(u^{\prime} g^{\prime}\right)\right|$ and $\left|\phi\left(u^{\prime \prime}\right)\right| \leq\left|s^{\prime \prime}\right|<\left|\phi\left(g^{\prime \prime} u^{\prime \prime}\right)\right|$. Define $t^{\prime}, t^{\prime \prime} \in \Sigma^{*}$ by $s^{\prime}=\phi\left(u^{\prime}\right) t^{\prime}$ and $s^{\prime \prime}=t^{\prime \prime} \phi\left(u^{\prime \prime}\right)$. Then

$$
\phi(u)=\phi\left(u^{\prime}\right) \phi\left(g^{\prime}\right) \phi\left(g^{\prime \prime}\right) \phi\left(u^{\prime \prime}\right)=\phi\left(u^{\prime}\right) t^{\prime}\left(t^{\prime \prime} \phi\left(u^{\prime \prime}\right),\right.
$$

so $\phi\left(g^{\prime}\right) \phi\left(g^{\prime \prime}\right)=t^{\prime} \ell t^{\prime \prime}$. By $\left|s^{\prime \prime}\right|<\left|\phi\left(g^{\prime \prime} u^{\prime \prime}\right)\right|$ we get $\left|t^{\prime \prime}\right|<\left|\phi\left(g^{\prime \prime}\right)\right|$. Define $\ell^{\prime \prime} \in \Sigma^{+}$by $\phi\left(g^{\prime \prime}\right)=\ell^{\prime \prime} t^{\prime \prime}$. Definc $\ell^{\prime} \in \Sigma^{*}$ by $\ell=\ell^{\prime} \ell^{\prime \prime}$. So $\phi\left(g^{\prime}\right)=t^{\prime} \ell^{\prime}$. By $\left|s^{\prime}\right|<\left|\phi\left(u^{\prime} g^{\prime}\right)\right|$ we get $\left|t^{\prime}\right|<\left|\phi\left(g^{\prime}\right)\right|$ and so $\ell^{\prime} \in \Sigma^{+}$.

Since $\phi\left(g^{\prime \prime}\right)$ is a prefix of $r$, we obtain $\ell^{\prime \prime} \in\left(\mathcal{L L}(\ell, r)\right.$, so $\ell^{\prime \prime}=\beta$ and $\ell^{\prime}=\ell_{i}$. Definc $h^{\prime}, h^{\prime \prime} \in \Omega \mathrm{by}$

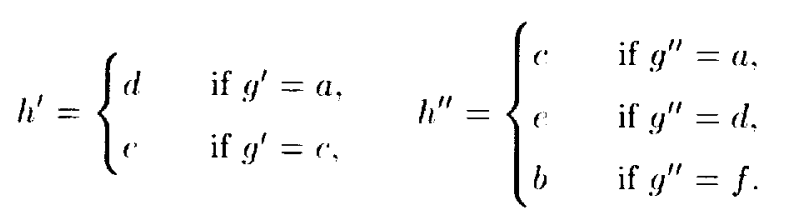

Then $g^{\prime} g^{\prime \prime} \rightarrow h^{\prime} f h^{\prime \prime}$ is in $R$, and moreover $\phi\left(g^{\prime}\right)=\phi\left(h^{\prime}\right) \ell_{, \beta}=t^{\prime} \ell_{\beta}$ and $\phi\left(g^{\prime \prime}\right)=\beta \phi\left(h^{\prime \prime}\right)=\beta t^{\prime \prime}$. So $t^{\prime}=\phi\left(h^{\prime}\right)$ and $t^{\prime \prime}=\phi\left(h^{\prime \prime}\right)$ and so

$$
t=s^{\prime} r s^{\prime \prime}=\phi\left(u^{\prime}\right) \phi\left(h^{\prime}\right) \phi(f) \phi\left(h^{\prime \prime}\right) \phi\left(u^{\prime \prime}\right)=\phi(v)
$$


for $v=u^{\prime} h^{\prime} f h^{\prime \prime} u^{\prime \prime}$. So $u \rightarrow k v$. By Le'mmal 6.3 we get $u \in$. M. Q

We are about to prove termination of $f \rightarrow r$ by a reduction to termuation of $R$. For this purpose we still need $\{t \rightarrow r\}$-reductions that start in $\phi[. \mathcal{M}]$. Such reductions are prosided by forward closures [10, 1] as we will show next. Wo use the following characterization of forward el swures by Hemann.

DIFINITON 6.6 ([6, Corollaire 2.16]). The set of for uard rlosures of a string rewriting rale $\rightarrow r$ oner alphabet $\Sigma$ is the least set $\mathrm{FC}(t \rightarrow r)$ of $(\rightarrow r$-reductions. such that

$f c l .(\ell \rightarrow r) \in \mathrm{F}(\mathrm{l}(l \rightarrow r)$.

fe. if $\left(s_{1} \rightarrow^{+} t_{1}^{\prime} \ell^{\prime}\right) \in \mathrm{FC}(\ell \rightarrow r)$ and $\ell^{\prime}=l^{\prime \prime \prime}$ for some $\ell^{\prime \prime}$. $\ell^{\prime \prime} \in \Sigma^{+}$then $\left(s_{1} f^{\prime \prime} \rightarrow^{+} t_{1}^{\prime} l^{\prime \prime \prime} l^{\prime \prime} t_{1}^{\prime} r\right) \in$ $\mathrm{FC}(t \rightarrow r)$.

fr:3. if $\left(s_{1} \rightarrow^{+} t_{1}^{\prime}\left(t_{1}^{\prime \prime}\right) \in \mathrm{FC}(r \rightarrow r)\right.$ then $\left(s_{1} \rightarrow^{+} t_{1}^{\prime}\left(t_{1}^{\prime \prime} \rightarrow^{+} t_{1}^{\prime} r t_{1}^{\prime \prime}\right) \in \mathrm{FC}^{\prime}(t \rightarrow r)\right.$.

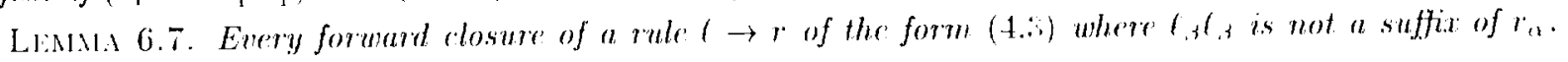
has a right hand side in $\phi[\mathcal{H}]$.

Proof. By induction along the definition of forward closure. Let $\left(s \rightarrow^{+} t\right) \in \mathrm{FC}(f \rightarrow r)$. In C'ase (fe1) we have $t=r=\phi(f)$. In Case (fo:3) the claim follows from Lemma 6.5. This leaves to prove (ase (fac2).

Suppose that $s=s_{1} f^{\prime \prime}, t=t_{1}^{\prime} r,\left(s_{1} \rightarrow^{+} t_{1}^{\prime} f^{\prime}\right) \in \mathrm{FC}(t \rightarrow r)$. and $t=f^{\prime} f^{\prime \prime}$ for some $f^{\prime \prime} f^{\prime \prime} \in \Sigma^{+}$. By inductive hypothesis, there is $u \in \mathcal{M}$ such that $t_{1}^{\prime} f^{\prime}=\phi(u)$. By definition of $\mathcal{M}, u$ has suftix $f$ or $f$ b.

Case 1: $"$ has suffix $f b$. Define $g^{\prime} \in \Omega^{*}$ by $u=g^{\prime} f b$. Then

$$
g^{\prime} \in\left(a+d\left(f^{\prime}\right)^{*}+d(f e)^{*} f(\cdot)^{*} d(f e)^{*}\right.
$$

by definition of $\mathcal{M}$. We distinguish cases whether $\left|f^{\prime}\right|>\left|r_{i 3}\right|$ or not.

Case 1.1: $\left|f^{\prime}\right|>\left|r_{i, 1}\right|$. The string $t_{1}^{\prime} f^{\prime}$ has suffix $\phi(f b)=r r_{\beta}$. By $|f|<|r|$ and $\left|f^{\prime}\right|>\left|r_{3}\right|$ we get $f^{\prime}=z r_{;}$ for some non-empty suffix $z$ of $r$. Now $z \in \operatorname{OVL}(r, \ell)$, so $z=$ a. So $t_{1}^{\prime} f^{\prime}=\phi\left(g^{\prime}\right) r r_{3}=\phi\left(g^{\prime}\right) r_{s} f^{\prime}$. whence $t_{1}^{\prime}=\phi\left(g^{\prime}\right) r_{a}=\phi\left(g^{\prime} a\right)$. So $t_{1}^{\prime} r=\phi\left(g^{\prime} a\right) r=\phi\left(g^{\prime} a f\right)$ for $g^{\prime} a f \in \mathcal{M}$.

Case 1.2: $\left|f^{\prime}\right| \leq\left|r_{i j}\right|$. Then $f^{\prime}$ is a suffix of $r_{i t}$ and so of $r$. So $l^{\prime} \in$ OVL $(r, f)$ whence $f^{\prime}=a$. So

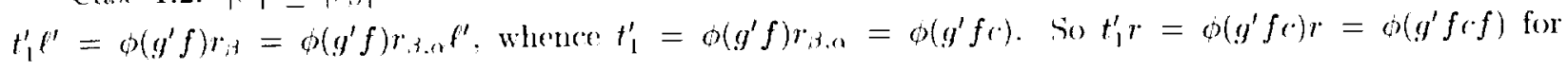
$g^{\prime} f \cdot f \in \mathcal{M}$.

Case 2: $u$ has suffix $f$. Define $g^{\prime} \in \Omega^{*}$ by $u=g^{\prime} f$. Then

$$
g^{\prime} \in\left(a+d\left(f()^{*}+d(f e)^{*} f c\right)^{*}\right.
$$

by definition of $\mathcal{M}$. By $|f|<|y|$ we get that $\ell^{\prime} \in$ OVL $(r . f)$, whence $\ell^{\prime}=\alpha$. So $t_{1}^{\prime} f^{\prime}=\phi\left(g^{\prime} f\right)=\phi\left(g^{\prime}\right) r=$ $\phi\left(g^{\prime}\right) r_{0} f^{\prime}$, whenee $t_{1}^{\prime}=\phi\left(g^{\prime}\right) r_{o}=\phi\left(g^{\prime} a\right)$. So $t_{1}^{\prime} r=\phi\left(g^{\prime} a\right) r=\phi\left(g^{\prime} a f\right)$ for $g^{\prime} a f \in \mathcal{M}$.

LEMMa 6.8. A rule $l \rightarrow r$ of the form (4.3) terminates if $\ell_{3} \ell_{3}$ is nut a suffix of $r_{n}$.

Proof. If $t \rightarrow r$ is non-terminating then there is an infinite rewriting seguence $s_{1} \rightarrow \rightarrow_{t \rightarrow r} s_{2} \rightarrow t \rightarrow r \ldots$ starting from a right hand side of a forward closure [1]. By Lemma $6.7 s_{1} \in \phi[\mathcal{M}]$. i.e., there is $u_{1} \in \mathcal{M}$ such that $\phi\left(u_{1}\right)=s_{1}$. By induction on $i$, using Lemma 6.5 , one casily proves that for every $i$ there is an $u_{i+1} \in \mathcal{M}$ such that both $u_{i} \rightarrow_{R} u_{i+1}$ and $\phi\left(u_{i+1}\right)=s_{i+1}$. Hence we get an infinite reduction secquence $u_{1} \rightarrow_{H} u_{2} \rightarrow_{H} \ldots$. Contradiction to termination of $R$. $\square$

EXAMPli: 2. For every $m \geq 0$, the one-rule $S R S$

$$
a b(d a b)^{m+1} a b \rightarrow d a b a b b(d a b)^{m-1} a
$$

is terminating by Lemma 6.8. With $m=0$ we get the smallest terminating witness $(|r|=10)$ of Lemma 4.4 .

This example also proves that kurth's [8] Criterion $F$ is incomplete. for Criterion $F$ applies only to the left barren or right barren cases [3. Theorem 6.31$]$. 
Wo note moreover that the maximal length of a deriation starting with $s \in \mathrm{S}^{*}$ is lincar in $|s|$. This is a direct consequence of the decreasing weight associated with a step $u \rightarrow_{n} v$.

7. The Main Theorem. Now we have all material together to prove our claim.

THEOREM 7.1. Let $|O \mathrm{~L}(r, f)|=|O \mathrm{OL}(\ell, r)|=1$. Then $\{\ell \rightarrow r\}$ terminates if and only if it has no loop of lengths 1 , 2. or 3.

Proof. Lot $O \mathrm{OVL}(r, \ell)=\{r\}$ and $O \mathrm{OLL}(\ell, r)=\{3\}$. If $\ell$ is a factor of $r$ then $\{\ell \rightarrow r\}$ has a loop of length $1[8]$. Else if $|\ell| \geq|r|$ then $\{\ell \rightarrow r\}$ terminates. If $\ell \rightarrow r$ is left barren or right barren then $\{f \rightarrow r\}$ terminates. So suppose that $f$ is not a factor of $r$; that $|\ell|<|r|$; and that $f \rightarrow r$ is neither left barren nor right barren. We distinguish cases:

Case $1: \ell \rightarrow r$ is neither left s-barren nor right s-barren. Then $r=r^{\prime} t_{3} \alpha$ and $r=\beta \ell_{a} r^{\prime \prime}$ for some strings $r^{\prime}, r^{\prime \prime}$. There is a loop of length 2 :

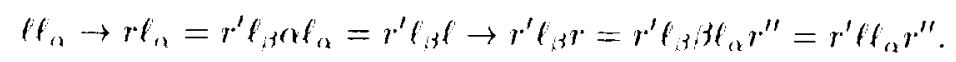

Case 2: $\ell \rightarrow r$ is left s-barren but not right s-barren. Then $f \rightarrow r$ has the form (4.3). If $\ell_{3} \ell_{3}$ is a suffix of $r_{a}$ then $\{f \rightarrow r\}$ has a loop of length 3 by Lemma 5.1 . Else $\{f \rightarrow r\}$ terminates by Lemma 6.8 .

Case 3: $l \rightarrow r$ is not left s-barren but right s-barren. This case is symmetric to Case $2:$ Wo have a loop of length 3 if $\ell_{r} \ell_{n}$ is a prefix of $r_{s}$, otherwise termination.

Case $4: f \rightarrow r$ is both left s-barren and right s-barren. Then Lemma 4.1 and its dual apply, showing $|\beta|>|\alpha|$ and $|\alpha|>|\beta|$, a contradiction. So this case does not exist. This finishes the proof.

Kurth [9] has proved decidability of the existence of loops of lengths 1, 2, or 3 for one-rule SRSs. Indeed. for every SRS and every $n \geq 1$, the existence of loops of lengths less or equal $n$ is decidable [5].

COROLLARY 7.2. Termination is decidable for one-rule SRSs $\{\ell \rightarrow r\}$ that satisfy $|\mathrm{OVL}(r, \ell)|=$ $\mid$ OVL $(\ell, r) \mid=1$.

8. Conclusion. We proved that termination of one-rule SRSs with one pair of overlaps is equivalent to the non-existence of loops of length less than or equal to 3 . Thus we showed that termination is decidable for one-rule SRSs with one pair of overlaps. A surprising observation in this investigation was the emergence of nom-tame rules, some admitting loops of length 3 , and some terminating. Such rules were not covered by the two precursor results by Kurth and by Shikishima-Tsuji et al.

Acknowledgements. Robert MeNaughton gave the author an appreciation of the intricacy of the problem.

\section{REFERENCES}

[1] N. Dershowitz, Termination of linear rewriting systems, in Proc. 8th Int. Coll. Automata, Languages and Programming. LNCS 115, Springer, 1981, pp. 448458.

[2] A. Geser, Decidability of termination of grid string rewriting rules, SIAM J. Comput., 31 (2002), pp. $1156 \cdot 1168$.

[3] _ Is termination decidable for string reuriting with only one rule? habilitation thesis, WilhelmSchickard-Institut, Universität Tübingen, Germany, Jan. 2002. 201 pages.

[4] _ Loops of superexponential lengths in one-rule string rewriting, in Proc. 13th Int. Conf. Rewriting Techniques and Applications. S. Tison, ed., LNCS 2378, Springer, 2002, pp. 267-280.

[5] A. Geser anb H. Zantema, Non-looping string rewriting. Theoret. Informatics Appl., 33 (1999), pp. $279-301$. 


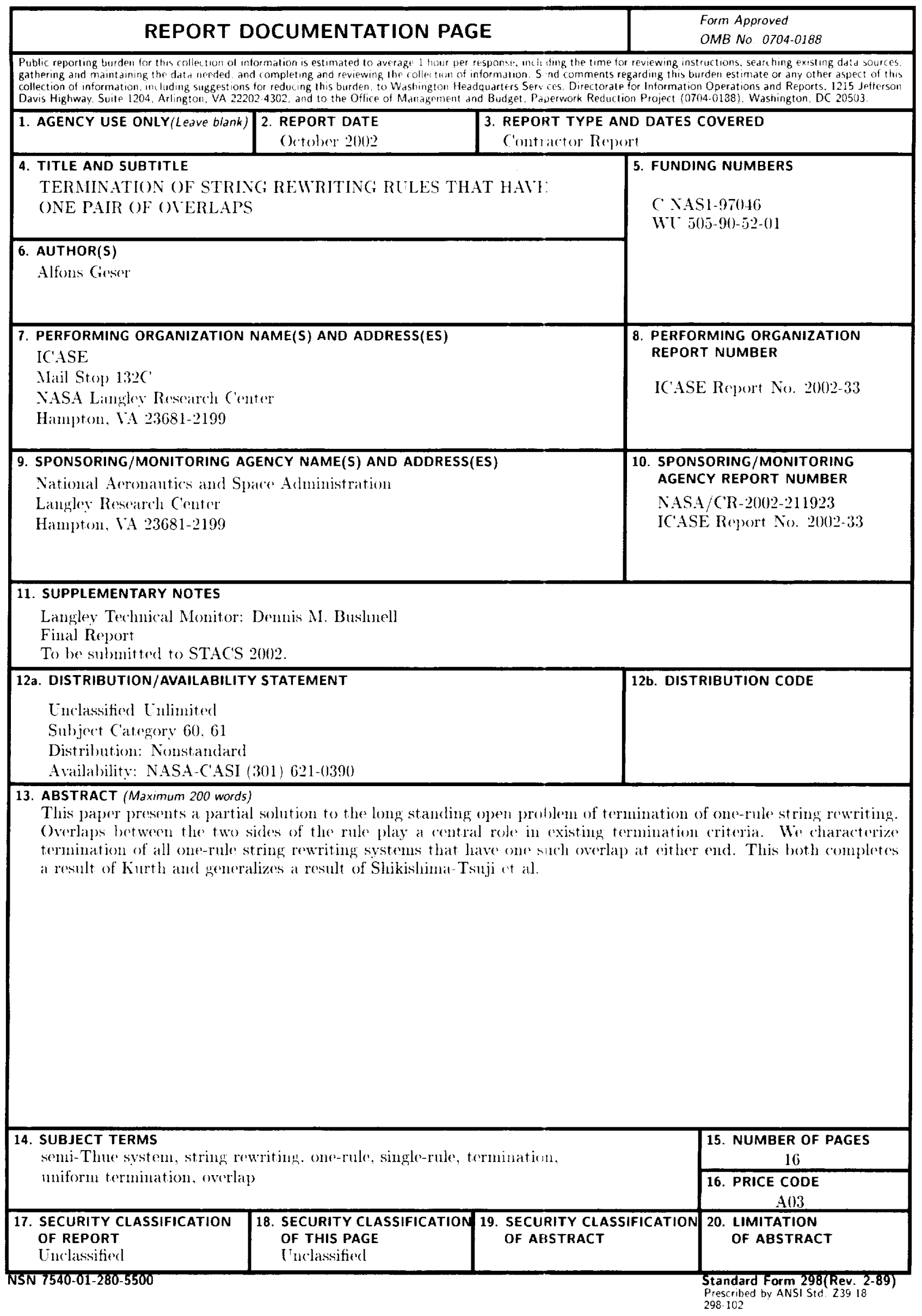


[6] M. Herman, Divergence des systèmes de récriture et schématisution des ensembles infinis de termes. habilitation, Université de Nancy, France, Mar. 1994.

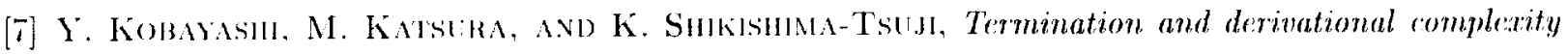
of confluent one-rule string rewriting systems, Theoret. Comput. Sci.. 262 (2001), pp). 583632.

[8] W. KunTII. Termination und Konfluenz won Semi-Thur-Systemen mit nur einer Regel, dissertation, Technische Universitait Clatusthal, Germany, 1990.

[9] __ One-rule semi-Thue systems with loops of length one, two, or three, RAIRO Inform. Theor., 30 (1995). p). 415429.

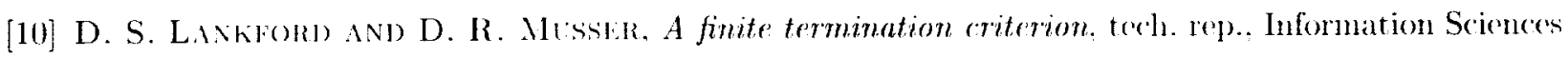
Institute, Cniv, of Southern California, Marina-del-Rey, CA. 1978.

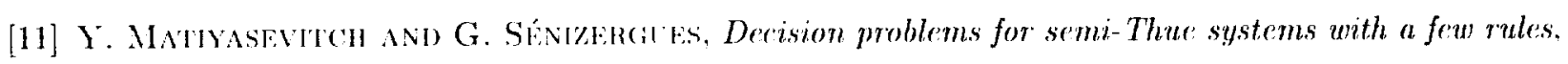
in Pror. 11th IEEE Symp. Logic in Computer Scionce, Now Brunswick, N.I, July 1996. IEEE Computer Society Press, pp. 523531.

[12] R. MCNAutiton. The uniform halting problem for one-rule Semi-Thue Systems. Tech. Rep. 94-18, Dept. of Computer Science, Rensselaer Polytechnic Institute, Troy, NY, Aug. 1994. See also "Correction to 'The Uniform Halting Problem for' One-rule Semi-Thue Systems'”, unpublished paper, Aug., 1996.

[13] _- Well-behaved derivations in one-rule Semi-Thue Systems, Tech. Rep. 95-15, Dept. of Computer Science, Rensselaer Polytechnic Institute, Troy, NY, Now 1995. See also "Correction by the author to "Well-behaved derivations in one-rule Semi-Thue Systems", unpublished paper, July, 1996.

[14] _. Semi-Thue Systems with an. Inhibitor, .J. Automated Reasoning, 26 (1997), pp. 409-431.

[15] G. SEvizercuts. On the termination problem for one-rule Semi-Thue Systems, in Proc. 7th Int. Conf. Rewriting Techniques and Applications, H. Ganzinger, ed., LNCS 1103. Springer, 1996, pp. 302316.

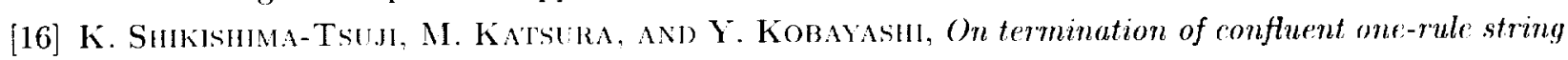
reuriting systems, Inform. Process. Lett., 61 (1997), pp. 9196.

[17] C. Wrathili. Confluence of one-rule Thue systems, in Word Equations and Related Topies, K. L. Schulz, ed., LNCS 572, Springer, 1992.

[18] H. Zantema and A. Geske, A complete chatucterization of termination of $0^{p} 1^{q} \rightarrow 1^{r} 0^{s}$, Applicable Algebra in Engineering, Communication, and Computing, 11 (2000), pp. 125. 


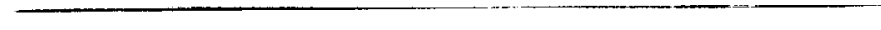


